\title{
Comparison of Two Corticosteroid Pre-Infusion Regimens for Pegloticase in the United States: A Retrospective Analysis in Community Rheumatology Practices
}

\author{
Amar Majjhoo $^{1}$ - Ada Kumar² Michael Zdanis $^{3} \cdot$ Brian LaMoreaux $^{2}$
}

Published online: 31 October 2019

(c) The Author(s) 2019

\begin{abstract}
Background Pegloticase is a recombinant porcine-like uricase enzyme that is FDA-approved for the treatment of chronic refractory gout in adults. Some patients receiving pegloticase develop anti-drug antibodies, which leads to both loss of pegloticase efficacy and an increased risk for infusion reactions. In the pivotal trials, all patients received pre-infusion medications before each pegloticase dose, including intravenous (IV) hydrocortisone. In clinical practice, many clinicians use methylprednisolone for pre-infusion therapy with pegloticase; however, the efficacy of methylprednisolone compared with hydrocortisone as a pre-infusion medication for pegloticase has not been established.

Objective The aim of this study was to compare the efficacy of methylprednisolone versus hydrocortisone as a pre-infusion medication for pegloticase.

Methods Data were retrospectively collected from 92 qualifying patients treated with pegloticase and administered preinfusion prophylaxis with either intravenous hydrocortisone or methylprednisolone. Patient demographics, steroid type and dose, duration of pegloticase therapy, overall number of infusions, and number of infusion reactions were assessed.

Results Patients treated with methylprednisolone as a pre-infusion medication received on average 8.5 pegloticase infusions versus 4.9 infusions for patients who were treated with hydrocortisone $(p<0.001)$. In addition, a significantly lower proportion of patients receiving methylprednisolone had their course of therapy terminated early due to infusion reactions $(8.2 \%)$ versus patients receiving hydrocortisone $(41.9 \%, p<0.01)$.

Conclusion In this retrospective chart-review project, patients were able to have a longer duration of pegloticase therapy, received a significantly greater number of infusions, and experienced fewer infusion reactions when methylprednisolone was used as the corticosteroid for pre-infusion prophylaxis compared with hydrocortisone.
\end{abstract}

Electronic supplementary material The online version of this article (https://doi.org/10.1007/s40801-019-00164-9) contains supplementary material, which is available to authorized users.

Ada Kumar

akumar2@horizontherapeutics.com

Amar Majjhoo

amarmajjhoo@hotmail.com

Michael Zdanis

mzdanis@cetusgroup.com

Brian LaMoreaux

blamoreaux@horizontherapeutics.com

Shores Rheumatology, St. Clair Shores, MI, USA

2 Horizon Therapeutics plc, 150 South Saunders Road, Lake Forest, IL 60045, USA

3 Cetus Group, LLC, Towson, MD, USA

\section{Introduction}

Uricase is an enzyme that degrades urate into a water-soluble form, allantoin. While most mammals possess uricase, humans lack this enzyme. The exact reason for the evolutionary loss of this enzyme in humans is not known. The lack of this innate enzyme predisposes humans to both hyperuricemia and accumulation of urate in the body, which may lead to gout.

Pegloticase is a mammalian recombinant uricase conjugated to polyethylene glycol (PEG) [1]. The compound was pegylated in order to prolong its half-life and potentially reduce immunogenicity [1]. Pegloticase rapidly metabolizes circulating uric acid into highly soluble allantoin, which is easily excreted in the urine. Pegloticase is an intravenously administered biologic therapy and is currently the only available, FDA-approved treatment for adult patients with chronic 


\section{Key Points}

Pegloticase is an effective therapy for lowering serum uric acid in patients with refractory gout. Some patients receiving pegloticase develop anti-drug antibodies, which leads to both loss of pegloticase efficacy and an increased risk for infusion reactions (IRs). This study retrospectively examined and compared pegloticase treatment parameters in refractory gout patients receiving pre-infusion prophylactic hydrocortisone or methyl-

Patients receiving pre-infusion methylprednisolone received more infusions, had a longer therapy duration, and had a lower rate of therapy discontinuation due to IRs than patients receiving pre-infusion hydrocortisone.

The efficacy of hydrocortisone in preventing pegloticaserelated IRs has been examined in clinical trials. However, many physicians standardly administer methylprednisolone in clinical practice. This study demonstrates that methylprednisolone may be more effective in preventing pegloticase therapy discontinuation than hydrocortisone. prednisolone.

Therefore, the use of pre-infusion prophylaxis is recommended with each pegloticase infusion to minimize the occurrence of IRs. During the RCTs, hydrocortisone $200 \mathrm{mg}$ IV in combination with antihistamines (oral fexofenadine $60 \mathrm{mg}$ ) and acetaminophen (1000 mg orally) were administered as the pre-infusion prophylaxis regimen.

Methylprednisolone has been given as a pre-infusion prophylaxis corticosteroid prior to the administration of other infusion biologic therapies, including infliximab and rituximab [7]. While hydrocortisone remains widely used by rheumatology practices as a pre-infusion prophylaxis for pegloticase, some providers use methylprednisolone as an alternative corticosteroid. The efficacy of methylprednisolone as a pre-infusion prophylaxis for pegloticase has not been previously systematically evaluated in the literature.

The purpose of this study was to perform a comparative analysis of intravenous methylprednisolone and intravenous hydrocortisone for pre-infusion prophylaxis in patients receiving pegloticase. This was done using a retrospective real-world dataset that included data on length of pegloticase therapy, number of infusions, and occurrence of IR. As corticosteroids are known to have immunomodulating properties, it is possible that different steroid exposures may have an effect on response to pegloticase and the occurrence of IR.

\section{Methods}

gout refractory to conventional therapy, or for whom conventional oral urate-lowering medications are contraindicated. In two replicate, blinded, randomized, placebo-controlled phase III trials (RCTs), the most common adverse event of pegloticase was gout flares reported in approximately $80 \%$ of patients. The second most common adverse event was infusion-related reactions occurring in $26 \%$ of patients with biweekly dosing, compared with $5 \%$ of patients in the placebo group [2, 3]. Infusion reactions (IRs) were defined as any infusion-related adverse event or cluster of temporally related events that occurred during or within $2 \mathrm{~h}$ after drug infusion, and that could not be reasonably attributed to another cause [4]. In the RCTs, serious IRs occurred in 5\% (biweekly dosing) of all patients [2]. Assessment of the IRs indicated the development of high-titer anti-drug antibodies was associated with a higher occurrence of IRs [5]. It is well-documented that elevated serum uric acid (sUA) levels immediately prior to pegloticase infusions while on therapy can reflect the development of anti-drug antibodies and therefore identify patients who may be at higher risk for IRs with continued treatment $[4,6]$. In the RCTs, investigators were blinded to sUA levels and patients were continued on pegloticase for the entire treatment duration regardless of their sUA levels. IRs were the most common reason for study discontinuation [2].

\subsection{Study Design and Patient Selection}

This study protocol was reviewed by the Western Institutional Review Board (Puyallup, WA, USA). Because of this study's retrospective nature, it was given exempt status, waiving the requirement of informed consent. All study conduct adhered to the tenets of the Helsinki Declaration of 1964, as revised in 2013, concerning human and animal rights.

Information detailing pre-infusion prophylaxis for pegloticase administration and resulting outcomes was collected from 19 geographically-dispersed, US community-based rheumatology practices between March 2015 and June 2016. Data from these participating practices was retrospectively collected for 96 patients beginning and ending on-label every-2-week dosing of pegloticase therapy between January 2013 and March 2016. Reasons for cessation of therapy included the following: decision by the prescribing clinician, termination request from the patient, and/or discontinuation due to loss of treatment response.

Standardized case report forms and data abstraction instructions were provided to participating sites with procedures for patient selection. To be eligible for inclusion in the study, patients must have met all of the following criteria: (i) 
have a primary diagnosis of gout (ICD-9 code 274.x); (ii) have been at least 18 years of age at the start of pegloticase therapy; (iii) have received their first infusion of pegloticase no earlier than January 1, 2013; (iv) have received at least two pegloticase infusions; and (v) have completed their course of pegloticase therapy. The dose and administration were per prescribing information $(8 \mathrm{mg}$ intravenous infusion over 120 min every 2 weeks). Delays and discontinuations in administration were noted for IR. No practices deviated from the prescribing information per normal treatment in the absence of an IR. Respondents blinded the identities of the patients through the use of anonymous numeric codes. As part of the chart review and data abstraction process, providers were asked to indicate if pegloticase therapy was discontinued prior to the completion of the planned treatment course. If this was true, the provider was further asked if the termination of therapy was due to patient request, loss of treatment response, and/or an IR. Upon receipt of the completed case report forms, the data aggregator subjected all data elements to range checks and identified outliers both within a submitting site and across participating sites. Queries were submitted to participating sites to resolve suspect data values.

\subsection{Data Elements and Operational Definition of Analysis Variables}

Data collected included patient demographics, duration of disease, prior urate-lowering therapy, sUA at baseline and prior to each pegloticase infusion, pre-infusion prophylaxis (including steroid type and dose), pegloticase infusion dates and times, pegloticase infusion length (in minutes), total number of pegloticase infusions, IR (classified as mild, moderate, or severe), and interventions.

Multiple ordinal logistic regression analysis was conducted. The primary dependent analysis variable was duration of therapy, defined as the number of infusions. Primary independent analysis variables were type of corticosteroid used for prophylaxis (hydrocortisone versus methylprednisolone), patient age (dichotomized as $<65 \mathrm{vs} \geq 65$ years), and terminal sUA (sUA as documented just prior to final infusion of pegloticase). Additional logistic regression analyses were conducted to examine the predictors of IR in this sample and to examine the effect of patient age on the duration of therapy.

\section{Results}

\subsection{Disposition and Patient Characteristics at Baseline}

Data from 96 patients were collected. Four patients were not included in the final analysis sample; three patients failed to meet study inclusion criteria, and one patient represented an extreme outlier due to the number of pegloticase infusions ( $>30$ infusions). The authors felt that it was appropriate to drop this patient from the analysis given the relatively small overall sample size. The analysis sample included a total of 670 pegloticase infusions. Exploratory analyses indicated that there were no significant differences in patient age, race, sex, disease duration, or sUA level prior to the initiation of pegloticase therapy between the two pre-infusion prophylaxis corticosteroid groups.

The demographic characteristics of the final analysis sample are summarized in Table 1 and are generally reflective of patients with chronic refractory/uncontrolled gout. The mean duration of gout was 13.8 years at the initiation of pegloticase therapy, and baseline sUA was $>6.8 \mathrm{mg} / \mathrm{dL}$.

The number of patients for each corticosteroid cohort and the dose mean and range of corticosteroid used for preinfusion prophylaxis are displayed in Table 2 . The mean dose of hydrocortisone used for pre-infusion prophylaxis was $198 \mathrm{mg}$, which is relatively consistent with the standard 200-mg pre-infusion prophylaxis dose used during the pegloticase RCTs. The range of doses for hydrocortisone was $150-200 \mathrm{mg}$. The standard dosing of methylprednisolone as a pre-infusion prophylaxis for pegloticase infusion has not been established in the literature. The mean dose of methylprednisolone used as a pre-infusion prophylaxis in this study was $77 \mathrm{mg}$ with a range of $40-120 \mathrm{mg}$. The steroid dose equivalent to $200 \mathrm{mg}$ of hydrocortisone is approximately $40 \mathrm{mg}$ of methylprednisolone. Therefore, the mean dose of methylprednisolone was notably higher than the equivalent mean dose of hydrocortisone.

\subsection{Regression Analysis}

In the regression analysis, use of methylprednisolone as a pre-infusion prophylaxis and terminal sUA were both highly significant predictors of therapy duration $(p<0.001$, Supplementary Table 1, see electronic Supplementary Material $[\mathrm{ESM}]$ ), as measured by the number of bi-weekly infusions. The use of methylprednisolone entered the model first as a predictor of therapy duration in this sample of patients.

In some patients, the doses of methylprednisolone administered were considerably greater than the equivalent corticosteroid dose of hydrocortisone. In order to examine the relative duration of therapy for the two pre-infusion regimens at more comparable dosages, a subset analysis was conducted that included only those methylprednisolone patients receiving doses of $60 \mathrm{mg}$ or less $(n=33)$. The results of this logistic regression analysis were similar to those of the entire methylprednisolone population. Use of lower doses of methylprednisolone as a pre-infusion steroid and terminal sUA both remained highly significant predictors 
Table 1 Demographic and clinical characteristics of refractory gout patients undergoing pegloticase therapy with a prophylactic pre-infusion corticosteroid

\begin{tabular}{llll}
\hline Characteristic & All patients $(n=92)$ & $\begin{array}{l}\text { Hydrocortisone patients } \\
(n=31)\end{array}$ & $\begin{array}{l}\text { Methylpredni- } \\
\text { solone patients } \\
(n=61)\end{array}$ \\
\hline $\begin{array}{lll}\text { Age (years) } \\
\text { Mean [SD] }\end{array}$ & $61.5[9.6]$ & $59.6[11.3]$ & $62.5[8.6]$ \\
Range & $38-77$ & $43-77$ & $38-77$ \\
Sex, $n(\%)$ & & & $47(77)$ \\
Male & $74(80)$ & $27(87)$ & $14(23)$ \\
Female & $18(20)$ & $4(13)$ & $49(80)$ \\
Race, $n$ (\%) & & & $5(8)$ \\
Caucasian & $73(79)$ & $24(77)$ & $5(8)$ \\
Black & $11(12)$ & $6(19)$ & $2(3)$ \\
Asian & $6(7)$ & $1(3)$ & $13.9[7.0]$ \\
Other & $2(2)$ & $0(0)$ & $4-30$ \\
Disease duration (years) & $13.8[6.7]$ & $13.5[6.2]$ & $8.5[2.5]$ \\
Mean [SD] & $4-30$ & $4-30$ & 517 \\
Range & & $4.9[2.7]$ & \\
Number of infusions & $7.3[3.1]$ & 153 & \\
Mean per patient [SD] & 670 & & \\
Total & & & \\
\hline
\end{tabular}

$S D$ standard deviation

Table 2 Pre-infusion prophylactic corticosteroid parameters administered to refractory gout patients undergoing pegloticase therapy

\begin{tabular}{lll}
\hline & Hydrocortisone & Methylprednisolone \\
\hline$n$ (patients) & 31 & 61 \\
Mean dose (mg) [SD] & $198.4[9.0]$ & $77.4[31.9]$ \\
Range (mg) & $150-200$ & $40-120$ \\
Modal dose (mg) & 200 & 50 \\
\hline
\end{tabular}

$S D$ standard deviation

of pegloticase therapy duration $(p<0.001$, Supplementary Table 2, see ESM).

In a binary logistic regression model, isolating the type of corticosteroid used for prophylaxis was a highly significant predictor of whether an IR occurred during pegloticase therapy $(p<0.001$, Supplementary Table 3A, see ESM). Both the use of methylprednisolone (odds ratio [OR] 0.13, 95\% confidence interval $[\mathrm{CI}]-3.3448$ to $-0.9212 ; p<0.001$ ) and age $\geq 65$ years (OR $0.27,95 \%$ CI -2.7236 to -0.0997 ; $p<0.05)$ appeared to protect against the development of an IR. However, when terminal sUA was introduced into the model, the type of corticosteroid used for pre-infusion prophylaxis did not remain a significant predictor of IR (Supplementary Table 3B, see ESM). This likely reflects the presence of anti-drug antibodies in patients on therapy who had elevated pre-infusion sUA levels ( $>6 \mathrm{mg} / \mathrm{dL}$ ).
Additional logistic models examining the relationship between age and duration of therapy were analyzed irrespective of corticosteroid used. A significant $(p<0.01)$ relationship between age and duration of therapy exists where age is examined as a linear predictor of duration of therapy both directly, and when dichotomized into the groups $<65$ years of age and $\geq 65$ years of age (Supplementary Tables $4 \mathrm{~A}$ and $4 \mathrm{~B}$, see $\mathrm{ESM}$ ). On average, patients $<65$ years of age received 1.8 fewer infusions compared with those $\geq 65$ years of age.

\subsection{Number of Infusions and Discontinuation of Therapy}

The mean number of pegloticase infusions for patients receiving methylprednisolone as prophylaxis was significantly higher than for patients receiving hydrocortisone as prophylaxis ( 8.5 vs $4.9 ; p<0.001)$. The range of the number of pegloticase infusions was 3-16 infusions for patients receiving hydrocortisone and 3-13 infusions for patients receiving methylprednisolone (Fig. 1).

The most frequent reason for premature discontinuation from the planned course of therapy was the development of an IR. All IR were classified as mild or moderate with symptoms similar to those noted during the pegloticase pivotal trials [3]. No anaphylactoid IRs were reported. As in the RCTs, when IR occurred, they were successfully managed 
by either stopping the infusion or continuing the infusion at a slower rate.

A significantly lower proportion of patients receiving methylprednisolone as prophylaxis had their course of therapy terminated early due to IR (8.2\%) as compared with patients receiving hydrocortisone as prophylaxis $(41.9 \%$; $p<0.01$; Fig. 2). In total, there were 13 IRs among 31 patients receiving hydrocortisone, all of which occurred at a dose of $200 \mathrm{mg}$. In contrast, five IRs were reported in 61 patients receiving methylprednisolone, of which two IRs occurred at a dose of $50 \mathrm{mg}$ and three IRs occurred at a dose of $100 \mathrm{mg}$.

In patients who completed the physician's planned course of pegloticase therapy, the mean number of infusions was greater than when therapy was terminated by the patient or when treatment was discontinued due to IRs, regardless of the type of steroid premedication administered $(p<0.01)$. For patients whose therapy was not discontinued, there was a significant difference between steroid groups in the mean number of infusions received ( 8.8 for methylprednisolone vs 5.9 for hydrocortisone; $p<0.01$ ). When therapy was discontinued, the mean number of infusions was not significantly different between groups ( 5 for methylprednisolone vs 3.6 for hydrocortisone; $p=0.18$ ), most likely due to the small number of patients discontinuing treatment in the methylprednisolone cohort. Cumulatively, for both outcomes, the mean number of infusions was greater for patients receiving methylprednisolone as prophylaxis (Fig. 3).

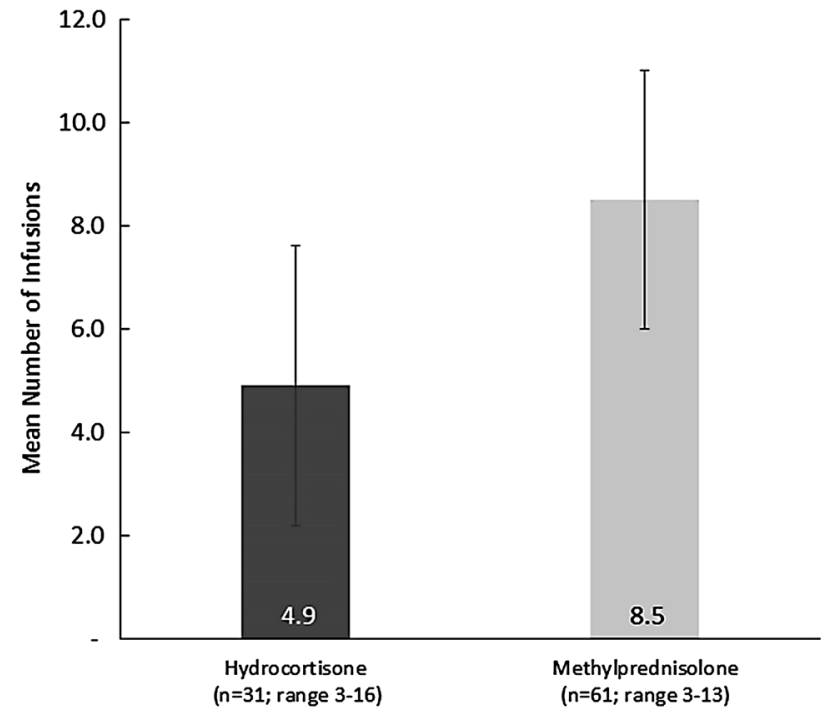

Fig. 1 Mean number of infusions in patients with refractory gout who were receiving pegloticase therapy and pre-infusion prophylactic glucocorticoids. Error bars represent one standard deviation

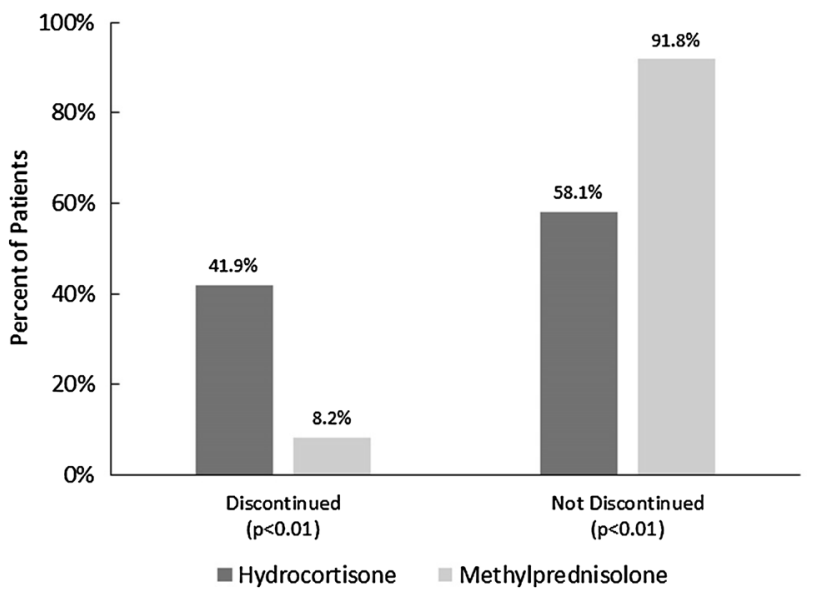

Fig. 2 Percent of refractory gout patients who discontinued pegloticase therapy. All patients were also receiving prophylactic pre-infusion glucocorticoids

\section{Discussion}

To the best of our knowledge, this study is the first systematic evaluation of methylprednisolone as an alternative to hydrocortisone as a pre-infusion corticosteroid prophylaxis for pegloticase. When methylprednisolone was used as a pre-infusion prophylaxis instead of hydrocortisone, patients in this retrospective study received a significantly greater number of pegloticase infusions and a significantly smaller proportion experienced IR. In this sample, patients administered methylprednisolone received, on average, a higher

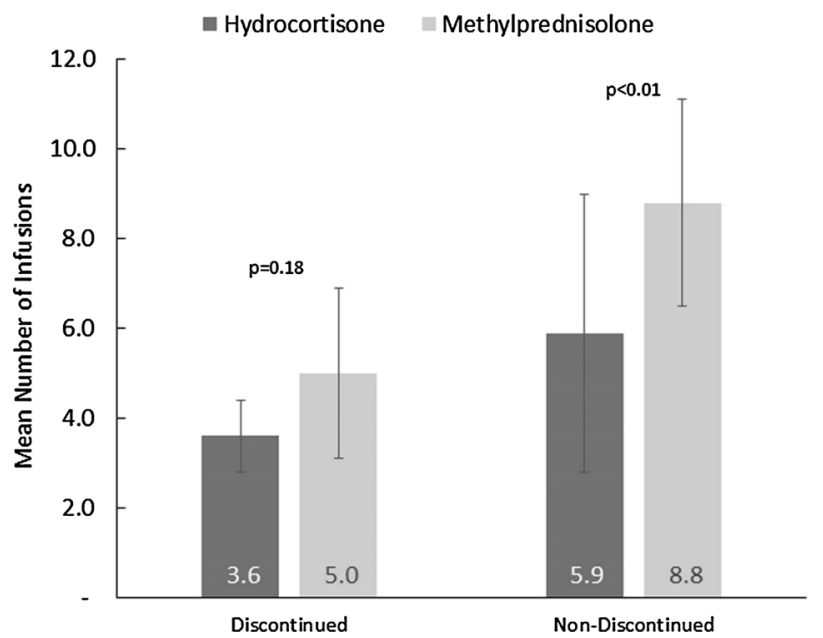

Fig. 3 Mean number of pegloticase infusions in refractory gout patients who discontinued and did not discontinue therapy. All patients were also receiving prophylactic pre-infusion glucocorticoids. Error bars represent one standard deviation 
dose equivalent of corticosteroid than the patients receiving hydrocortisone. Additionally, the higher doses of glucocorticoid may suppress some IR symptoms. In addition, the higher mean doses of methylprednisolone may have caused a greater immunomodulatory effect than the lower dose equivalent of hydrocortisone, thus attenuating the immunogenicity of pegloticase. Immunogenicity involving the development of anti-drug antibodies to pegloticase remains a major risk factor for development of IRs and discontinuation of therapy [5]. However, based on subgroup analyses, lower doses of methylprednisolone still imparted a beneficial effect on therapy duration.

In an effort to generate more controlled data around this concept $[8,9]$, studies examining how disease-modifying anti-rheumatic, immunomodulator therapies may improve pegloticase response rates are currently underway utilizing methotrexate (MIRROR trial, NCT03635957) [10], mycophenolate mofetil (RECIPE trial, NCT03303989), and azathioprine (TRIPLE trial, NCT02598596).

The current project depicted current usage of two different intravenous corticosteroids for pre-infusion prophylaxis for pegloticase therapy in a convenience sample of US community rheumatology practices. Limitations of this study include its small sample size, which necessitated the elimination of a single outlier from the study. In addition, this study did not capture possible long-term glucocorticoidrelated adverse events, especially in the setting of higher corticosteroid dose equivalents used with methylprednisolone than with hydrocortisone. The retrospective nature of the study is also a limitation. Finally, the development of anti-pegloticase antibodies in patients who experienced IRs was not measured in this analysis. While this study did show statistically significant benefits of methylprednisolone as a pre-infusion corticosteroid for pegloticase compared with hydrocortisone, a further dose-controlled prospective study to evaluate the effect of varying pre-infusion steroids on both efficacy and long-term safety is needed to verify the results.

\section{Conclusions}

In this evaluation, patients receiving methylprednisolone compared with hydrocortisone as a pre-infusion prophylaxis for pegloticase encountered fewer infusion reactions and were able to receive a greater number of doses of pegloticase infusions. This finding suggests that choice and dose of pre-infusion corticosteroid therapy can impact response to pegloticase in uncontrolled gout patients.

Data Availability The datasets generated during and/or analyzed during the current study are available from the corresponding author on reasonable request.

\section{Compliance with Ethical Standards}

Funding Sponsorship for this study and article processing charges were funded by Horizon. All authors had full access to all of the data in this study and take complete responsibility for the integrity of the data and accuracy of the data analysis.

Conflict of interest B. LaMoreaux and A. Kumar are employees of, and have stock in Horizon. A. Majjhoo has received consulting and speaking fees from Horizon. M. Zdanis has received consulting fees from Horizon.

Ethical approval All procedures performed in studies involving human participants were in accordance with the ethical standards of the institutional and/or national research committee and with the 1964 Helsinki Declaration of 1964 and its later amendments or comparable ethical standards. This study protocol was reviewed by the Western Institutional Review Board (Puyallup, WA, USA) and assigned exempt status, waiving the requirement of informed consent.

Authorship All named authors meet the International Committee of Medical Journal Editors (ICMJE) criteria for authorship for this article, take responsibility of the integrity of the work as a whole and gave given their approval for this version to be published.

Medical Writing, Editorial, and Other Assistance Editorial assistance in the preparation of this article was provided by Megan Francis-Sedlak, $\mathrm{PhD}$ of Horizon Therapeutics plc.

Open Access This article is distributed under the terms of the Creative Commons Attribution-NonCommercial 4.0 International License (http://creativecommons.org/licenses/by-nc/4.0/), which permits any noncommercial use, distribution, and reproduction in any medium, provided you give appropriate credit to the original author(s) and the source, provide a link to the Creative Commons license, and indicate if changes were made.

\section{References}

1. Sundy JS, Ganson NJ, Kelly SJ, et al. Pharmacokinetics and pharmacodynamics of intravenous PEGylated recombinant mammalian urate oxidase in patients with refractory gout. Arthritis Rheum. 2007;56:1021-8.

2. Sundy JS, Baraf HS, Yood RA, et al. Efficacy and tolerability of pegloticase for the treatment of chronic gout in patients refractory to conventional treatment: two randomized controlled trials. JAMA. 2011;306:711-20.

3. Guttmann A, Krasnokutsky S, Pillinger MH, Berhanu A. Pegloticase in gout treatment-safety issues, latest evidence and clinical considerations. Ther Adv Drug Saf. 2017;8:379-88.

4. Baraf HS, Yood RA, Ottery FD, Sundy JS, Becker MA. Infusionrelated reactions with pegloticase, a recombinant uricase for the treatment of chronic gout refractory to conventional therapy. J Clin Rheumatol. 2014;20:427-32.

5. Lipsky PE, Calabrese LH, Kavanaugh A, et al. Pegloticase immunogenicity: the relationship between efficacy and antibody development in patients treated for refractory chronic gout. Arthritis Res Ther. 2014;16:R60.

6. Keenan RT, Baraf HSB, LaMoreaux B. Use of pre-infusion serum uric acid levels as a biomarker for infusion reaction risk in patients on pegloticase. Rheumatol Ther. 2019;6:299-304. 
7. Picoraro J, Winberry G, Siegel CA, et al. Premedication use before infliximab administration: a cross-sectional analysis. Inflamm Bowel Dis. 2017;23:174-80.

8. Berhanu AA, Krasnokutsky S, Keenan RT, Pillinger MH. Pegloticase failure and a possible solution: immunosuppression to prevent intolerance and inefficacy in patients with gout. Semin Arthritis Rheum. 2017;46:754-8.

9. Freyne B. A case report of immunosuppressant medication associated polyarticular tophaceous gout successfully treated using the polyethylene glycolconjugated uricase enzyme pegloticase. Transpl Proc. 2018;50:4099-101.

10. Botson J, Peterson J. Pretreatment and coadministration with methotrexate improved durability of pegloticase (Krystexxa) response: a prospectve, proof-of-concept case series. Arthritis Rheumatol. 2018;70:1408. 